Repetitive transcranial magnetic stimulation (rTMS) is a tool with antidepressant potential that uses a coil placed on the scalp to produce a powerful magnetic field that directly stimulates only the outermost cortex. MRI scans were obtained in 29 depressed adults involved in an rTMS antidepressant clinical treatment. These scans were analyzed to investigate the effect of distance from coil to cortex on clinical parameters. Longer motor cortex distance, but not prefrontal distance, strongly correlated with increased motor threshold $(\mathrm{P}<0.01)$. Clinical antidepressant response did not correlate with either distance. The rTMS antidepressant responders, however, were significantly younger $(\mathrm{t}=-2.430, \mathrm{P}<0.05)$, and there appears to be a maximum threshold of age and distance to prefrontal cortex for response.

(The Journal of Neuropsychiatry and Clinical Neurosciences 2000; 12:376-384)

\section{How Coil-Cortex Distance Relates to Age, Motor Threshold, and Antidepressant Response to Repetitive Transcranial Magnetic Stimulation}

\author{
F. Andrew Kozel, M.D. \\ Ziad Nahas, M.D. \\ Cart deBrux, B.S. \\ Monica Molloy, M.S.N. \\ Jeffrey P. Lorberbaum, M.D. \\ Daryl Bohning, Ph.D. \\ S. Craig Risch, M.D. \\ Mark S. George, M.D.
}

Repetitive transcranial magnetic stimulation (rTMS) Ris a new tool to study brain function and is being investigated as a treatment modality for depression and other neuropsychiatric disorders. ${ }^{1,2}$ To perform rTMS, a powerful electromagnetic coil is placed on the scalp. The coil produces a rapidly changing focal magnetic field that induces an electrical current that depolarizes neurons. Because the magnetic field produced by the coil decreases exponentially with distance, ${ }^{3}$ only superficial structures are directly stimulated.

Currently in most rTMS applications, rTMS is dosed for each individual according to the amount of stimulation required to cause contraction of the contralateral (right extremity) abductor pollicis brevis. This is called the motor threshold (MT), and it is commonly expressed as a percentage of the total magnetic pulse capable for each machine. Surprisingly, despite more than a decade of research now using TMS as a tool to investigate the motor system, the relationship between the motor threshold for each individual and the distance from the individual's scalp to cortex is not well understood. Be-

Received August 24, 1999; revised January 12, 2000; accepted January 27, 2000. From the Functional Neuroimaging Division and the Brain Magnetic Stimulation Laboratories, Departments of Psychiatry, Radiology, and Neurology, Medical University of South Carolina; and Psychiatry Department, Ralph H. Johnson VA Medical Center, Charleston, South Carolina. Address correspondence to Dr. Kozel, MUSC Psychiatry Department, 67 President Street, P.O. Box 250861, Charleston, SC 29425; e-mail: kozelfa@musc.edu

Copyright (C) 2000 American Psychiatric Press, Inc. 
cause the motor threshold is inexpensively determined and appears to relate to seizure risk, ${ }^{4}$ most studies using TMS over nonmotor regions such as the prefrontal cortex have stimulated with the intensity determined by the motor threshold over motor cortex. An untested assumption is made that the motor cortex variables apply to the prefrontal cortex.

Initial open studies ${ }^{5-7}$ and later crossover ${ }^{8,9}$ and now double-blind parallel studies ${ }^{10}$ all suggest that rTMS has antidepressant properties. Not all studies have been positive. ${ }^{11}$ For example, psychotically depressed patients appear not to respond to rTMS as currently applied. ${ }^{12}$ Moreover, in all studies, older subjects have not responded as well as younger subjects. ${ }^{7,13}$

Imaging studies have shown that the prefrontal cortex atrophies with age in depressed subjects. ${ }^{14,15}$ Accordingly, the trend of nonresponse in elderly patients in the TMS antidepressant trials prompted us to wonder if the degree of brain atrophy, particularly prefrontal, might play a role in the relative nonresponse in older depressed subjects. We thus carried out the following magnetic resonance imaging (MRI) study in adult depressed subjects participating in a parallel-design randomized placebo-controlled trial of left prefrontal rTMS for the treatment of depression. (For full details of this clinical trial, see Nahas et al. ${ }^{10}$ )

\section{METHODS}

\section{Subjects}

Thirty-two depressed adults enrolled in a 2-week doubleblind placebo-controlled trial. Two subjects who had been randomized to receive active rTMS did not tolerate the procedure and dropped out after fewer than three treatments. They were excluded from final analysis. Prior to treatment, all subjects had an MRI scan of the head. One subject could not tolerate the initial MRI scan. Included for this MRI study, therefore, were 29 patients (11 men) who met DSM-IV criteria for either major depressive disorder $(n=21 ; 7$ men) or bipolar disordermost recent episode depressed ( $n=8 ; 4$ men). They were randomized into one of three cells, in each of which the subjects received 10 days of prefrontal stimulation over 2 weeks. The cells and the subject means $( \pm S D)$ for Hamilton depression score (Ham-D) and years of age were as follows: high frequency (Active, $20 \mathrm{~Hz}, n=12$; Ham- $\mathrm{D}=30 \pm 5.86$, age $=42.6 \pm 14$ ); low frequency (Active, $5 \mathrm{~Hz}, n=8 ; \mathrm{Ham}-\mathrm{D}=26.3 \pm 5.98$, age $=42.4 \pm 7$ ); and placebo $(n=9$; Ham- $\mathrm{D}=23.8 \pm 4.1$, age $=48.5 \pm 8.8)$. Following full explanation of the procedures, all subjects signed a written informed consent document according to the declaration of Helsinki and as approved by the
Medical University of South Carolina Institutional Review Board and the U.S. Food and Drug Administration Devices Section.

Subjects were free of antidepressant medications for at least 2 weeks prior to study entry. Three exceptions were bipolar patients who required ongoing mood stabilizers or benzodiazepines for anxiety. No subjects were currently abusing substances. Two subjects, however, had a history of alcohol dependence, and one had a history of heroine and butane abuse.

Subjects underwent an MRI scan of the brain at the beginning and end of the study ${ }^{16}$ and had three regional cerebral blood flow single-photon emission computed tomography (SPECT) scans: at baseline, during the fifth rTMS session, and 3 days after completion of the trial prior to restarting medications. This report discusses the initial MRI scans only; the SPECT results have been discussed elsewhere. ${ }^{17,18}$ Qualitative and quantitative analysis of MRI scans done before and after the 2 weeks of treatment showed that rTMS produced no MRI changes. ${ }^{16}$

\section{Transcranial Magnetic Stimulation}

A medical doctor (A.M.S., Z.N., or M.S.G.) trained in the proper use of repetitive rTMS used a Cadwell Magnetic Stimulator (Cadwell; Kennewick, WA) equipped with a figure-8-shaped coil and a continuous water cooling system to prevent overheating. Subjects were seated upright in a comfortable chair with eyes open during rTMS. On the initial treatment visit, motor threshold was determined at rest in the contralateral (right extremity) abductor pollicis brevis (APB) muscle, as described previously, ${ }^{13}$ by using visible twitch. The left prefrontal cortex stimulation site was defined as the location $5 \mathrm{~cm}$ rostral and in a parasagittal plane from the site of maximal APB stimulation.

Subjects were randomly assigned to receive stimulation over 20 minutes each weekday morning for 2 weeks as active $\left(5 \mathrm{~Hz}\right.$ or $20 \mathrm{~Hz}$; see Nahas et al. ${ }^{10}$ for discussion of the role of stimulation frequency) or placebo (coil held tangential off the head). The left prefrontal cortex was stimulated at $100 \%$ MT, with an equal total number of 16,000 stimulations across all cells.

\section{Ratings and Response Classification}

Before entering the study, subjects were screened and diagnosed by trained clinicians using the Schedule for Affective Disorders and Schizophrenia. ${ }^{19}$ In addition, the Ham-D (21 items ${ }^{20}$ was administered at baseline, on the fifth day of treatment (end of week 1), and at the end of the study (week 2). Trained psychiatric nurses, blind to treatment arm, performed all ratings. Ham-D scores were used to calculate percentage improvement 
from the beginning to the end of treatment (2 weeks). Subjects who showed $\geq 50 \%$ improvement in the 21 -item Ham-D at 2 weeks from baseline were classified as antidepressant treatment responders.

\section{MRI Scanning}

One day prior to the beginning of treatment, a $\mathrm{T}_{1}$ weighted $3 \mathrm{D}$ volumetric MRI sequence was obtained with a 1.5-tesla Picker MRI scanner (Picker International; Cleveland, OH). Scans in this study were 142 1mm-thick sagittal slices covering the entire brain $(128 \times 128, \mathrm{FOV}=20 \mathrm{~cm}, \mathrm{TE}=4.4, \mathrm{TR}=13$; voxel size $1.2 \times 1.2 \times 1 \mathrm{~mm}$ ). Most, but not all, subjects (21 of 29) had vitamin $\mathrm{E}$ capsule fiducials (approximately 7 $\mathrm{mm} \times 12 \mathrm{~mm}$ oval) placed at the site of rTMS prefrontal stimulation.

\section{MRI Scan Reformatting and Distance Calculations}

The $\mathrm{T}_{1}$-weighted MRI scans were reformatted from sagittal to coronal plane by a blinded reader (F.A.K. or C.D.) using Analyze Mayo Clinic Image Processing System version 7.5.2 on a SUN UltraSPARC 20 station. A line was drawn on the midsagittal view to bisect the anterior commissure and posterior commissure. The corresponding transverse image was resliced and corrected for problems in roll or yaw. The roll-correction was done by equalizing the structures of both eyes in the transverse plane and correcting yaw such that the midsagittal sulcus appeared vertical in that slice. The image was reformatted to the coronal plane, with 2501 -mm-thick slices from the caudal to the rostral aspect of the skull.

MRI distance measurements were performed by a trained observer (F.A.K.) blind to all patient variables, using MedX 2.1 (Sensor Systems; Alexandria, VA) on a DEC Alpha workstation with a 21-inch high-resolution screen. The coronal MRI images were enlarged so that there were three slices per screen (approximately $7 \times 12$ $\mathrm{cm}$ image size on the screen). The software, MedX, has a semi-automated function where, when a line is drawn from one point to another, the distance (in $\mathrm{mm}$ ) and a reference angle (e.g., moving from one point vertically upward to another gives a value of -90.00 degrees) are computed based on image voxel sizes (see Figure 1).

\section{Prefrontal Cortex Distance Measurement (D-PFC)}

A trained and blinded investigator (F.A.K.) assessed the shortest distance from the TMS coil to the nearest prefrontal cortex by using three different techniques.

1. Fiducial Method. The first measuring method utilized the central eight contiguous coronal scans where the prefrontal fiducial was visible. The distance from the proximal center edge of the fiducial to the nearest cortex in the coronal plane was measured in millimeters.

2. Modified Fiducial Method. Because of concerns regarding movement of the fiducial off of the scalp by gravity or hair, the second method measured the same scans from the outer skull directly under the middle of the fiducial to the nearest cortex.

3. Standardized Method. The fiducial was not used for these measurements. To determine which scans would be measured, the first scan anterior to the corpus callosum was identified. After moving anteriorly 16 scans $(16 \mathrm{~mm})$, we measured this scan and the seven subsequent scans. Taking a point on the central lateral edge of the left eye, a -90-degree line was drawn to the skull. The distance from the point of the line intersecting the outer skull to the nearest cortex was measured. In most subjects, this standardized method also intersected the fiducial. Nine subjects showed an overlap on most slices of the standardized and fiducial methods, and an additional 4 subjects had the fiducial within $5 \mathrm{~mm}$ of the spot determined by the standard method $(13 / 21$ or $62 \%)$. In $38 \%(8 / 21)$, the fiducial was caudal, rostral, or displaced off the skull. This standard method proved more reliable and also allowed for data analysis in an additional 8 subjects where the fiducial was not visible on the MRI scan $(21+8=29$ total scans). Performing this measurement on the $\mathrm{T}_{1}$ weighted MRI template scan in Talairach space resulted in a "fiducial region" extending in the $x$ plane from -25 to $-40, y$ from 50 to 58 , and $z$ from 20 to 40. Unfortunately, the prefrontal cortex distance on common Talairach templates could not be measured because of the lack of skull anatomical landmarks in the Talairach template scan.

After determining the D-PFC with all three methods, we elected to use the standard method for testing our hypotheses because it allowed the inclusion of the 8 subjects where the fiducial was not visible. In addition, there were concerns that the fiducials, even when visible, might have been moved from the true site because of hair, gravity, or displacement by the inflatable head holder used while scanning.

\section{Motor Cortex Distance Measurement (D-MC)}

Fiducials were not placed at the scalp position where the motor cortex threshold was determined. Because of the difficulty of determining the motor cortex region for thumb control from a structural MRI scan, particularly in the coronal plane, ${ }^{21}$ we chose not to identify the motor cortex directly. In the clinical trial, however, we determined the prefrontal stimulation location by empirically 
finding the area of motor cortex for $\mathrm{APB}$ and then moving in a parasagittal plane $5 \mathrm{~cm}$ forward. Thus, the motor cortex stimulation site would theoretically be $5 \mathrm{~cm}$ caudal from the prefrontal stimulation site. We therefore decided to use the prefrontal site as the point of refer- ence from which to determine the motor cortex site, using the following algorithm.

In order to measure the distance from the coil to the motor cortex, we first counted 16 slices rostral from the corpus callosum (which was the first slice measured us-

FIGURE 1. MRI scans demonstrating the different measurements described in this study. Panel A: The midline sagittal scan demonstrates the anterior commissure (ac) to posterior commissure (pc) line that was used to reformat all slices in the coronal plane (Panels B-D). In Panel A, the vertical line B shows the range where motor cortex measurements were taken (also seen in the coronal slice Panel B); the vertical line $\mathrm{C}$ demonstrates the region where the prefrontal measurements were taken (also seen in the coronal slice Panel C); and the vertical line A is the anterior slice of the corpus callosum that was used to determine the prefrontal region. Panel $B$ shows the measurement of the motor cortex; Panel $C$, the measurement of the prefrontal cortex. Panel D shows one of the subjects in the study with prominent prefrontal atrophy. On panels B-D, the right side of the image is the patient's left. The fiducials are clearly seen on $C$ and $D$ over the left prefrontal cortex, and the lines for distance measurements are indicated.
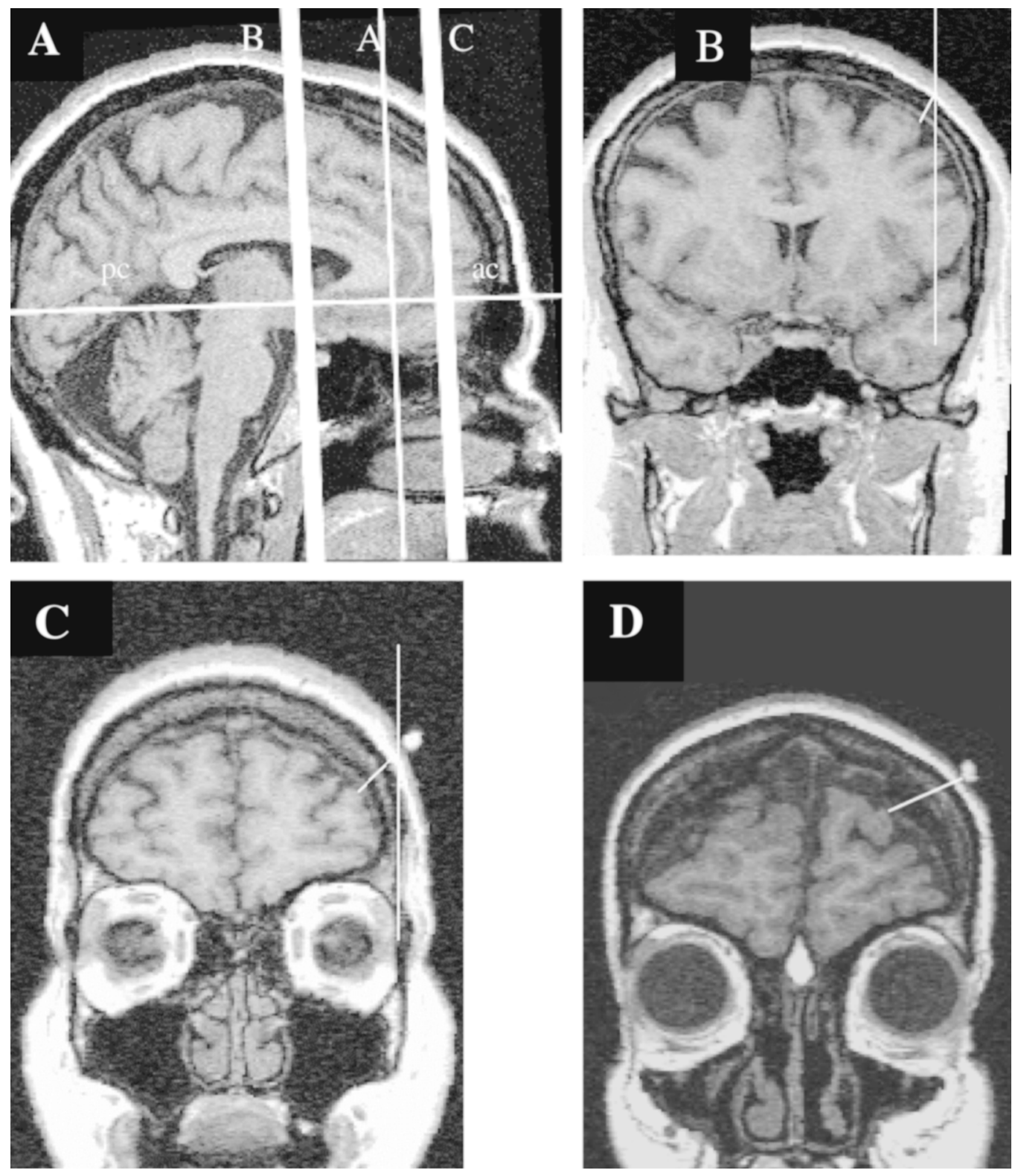
ing the standard method) and copied the vertical line from lateral left eye socket to the skull that was created by the standard method for measuring the prefrontal cortex. We then moved 50 slices $(5 \mathrm{~cm})$ caudally, and the vertical line was copied unchanged onto this slice (the first presumed motor cortex slice). This provided a point of intersection between the skull and the line. A measurement was taken from that intersection to the nearest cortex. This was recorded as the distance from the coil to the motor cortex in millimeters (D-MC). The line was copied onto the next eight rostral contiguous slices, and distance measurements were taken as above.

\section{Statistical Analysis}

First, using a paired Student's t-test, we compared whether D-MC significantly differed from D-PFC. Correlational hypotheses were tested by using StatView 4.5, where bivariate plots were performed and a Pearson's correlation with a Fisher's $r$ to $z$ ( $P$-values) was calculated to determine if significant relationships existed between our hypothesized variables $(P<0.05)$.

We analyzed the relationship of distance from coil to motor cortex (D-MC) with 1) percentage output to reach motor threshold; 2) age; 3) distance from coil to prefrontal cortex (D-PFC); and 4) percentage antidepressant clinical response. Next, we analyzed the correlation of D-PFC with 1) percentage clinical response; 2) age among responders and nonresponders; and 3) percentage output to reach motor threshold. Finally, potential relationships between the nondistance measures were tested (age, percentage output to reach motor threshold, and percentage antidepressant clinical response).

\section{RESULTS}

\section{Subjects}

For a full description of this patient sample, see Nahas et al. ${ }^{10}$ As noted above, there were 11 men and 18 women; 20 subjects with MRI scans were in the active group and 9 in the placebo group. This antidepressant trial had 7 responders and 22 (9 placebo) nonresponders.

\section{Motor Distance and Prefrontal Distance}

D-PFC was significantly greater than D-MC (D$\mathrm{PFC}=14.4 \pm 2.7 \mathrm{~mm}, \mathrm{D}-\mathrm{MC}=12.7 \pm 2.6 \mathrm{~mm}, t=-3.6$, $P<0.01$; mean \pm SD and Student's paired $t$-test).

Distance and Motor Threshold

MT significantly increased with increasing D-MC $(P<0.01$, Fisher's $r$ to $z, 29$ subjects; see Figure 2). There was no significant relationship between D-PFC and MT
$(P=0.0525)$. D-MC and D-PFC significantly crosscorrelated $(r=0.562, P<0.01)$.

\section{Correlation with Age}

D-MC $(r=0.525, P<0.01)$ as well as D-PFC $(r=0.611$, $P<0.001)$ significantly increased with age. Interestingly, a trend for D-PFC to increase more with age than D-MC was found in this depressed cohort (see Figure 3). In this small group, there was not a significant correlation $(P=0.5746)$ between age and D-MC minus D-PFC. There was not a significant correlation between age and MT $(P=0.1340)$ or between age and percentage antidepressant response $(P=0.1271)$.

\section{Correlation with Clinical Antidepressant Response}

There was no correlation between D-MC or D-PFC and percentage clinical response. (D-PFC did not correlate with percentage clinical response with any of the three measuring methods: fiducial to cortex $(P=0.1216)$, skull under fiducial to cortex $(P=0.4885)$, or standard $(P=0.2029)$. When we examined D-PFC and age, analyzed separately for responders and nonresponders (see Figure 4), the responders were significantly younger $(t=-2.430, P=0.0258)$, but response did not significantly correlate with D-PFC. There does seem to be a maximum threshold of age and distance, with the responders being younger than 55 years of age and having a D-PFC of less than $17.00 \mathrm{~mm}$.

There was not a significant correlation between percentage output to reach MT and percentage clinical antidepressant response $(P=0.1693)$.

FIGURE 2. Motor cortex distance (MC) versus motor threshold. For all subjects, motor threshold increases as the distance from skull to motor cortex increases.

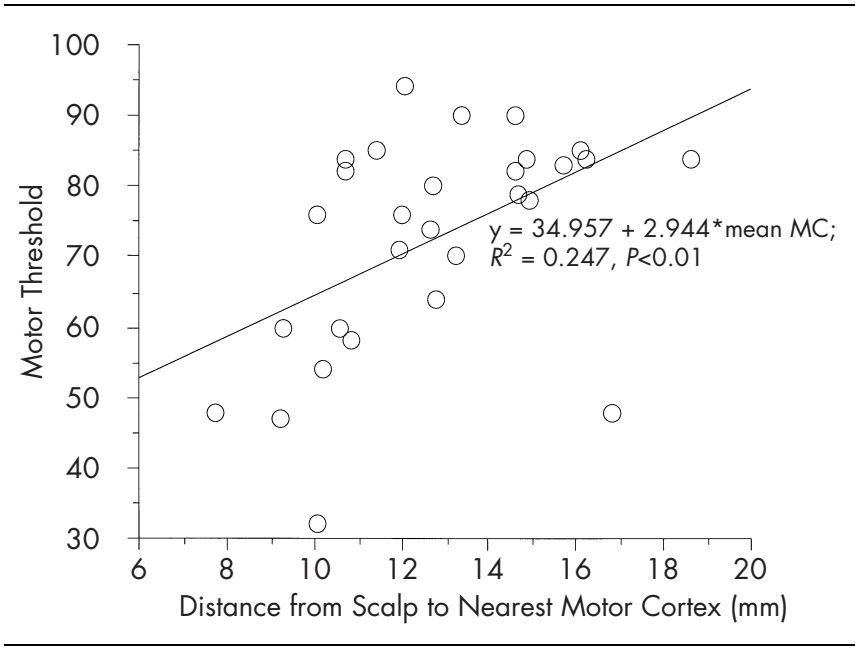




\section{DISCUSSION}

This is the first study addressing the complicated area of whether and to what degree the distance from coil to motor or prefrontal cortex interacts with motor threshold. This study also examines the relationships between age, prefrontal cortex distance, and clinical antidepressant response. As the first study exploring these questions, it suffers from several methodological shortcomings outlined below. Nevertheless, there were several important findings. The estimated distance from TMS coil to prefrontal cortex (D-PFC) was greater than the distance from coil to motor cortex (D-MC). The motor threshold (MT) significantly correlated with D-MC, whereas it did not correlate with D-PFC. Both motor and prefrontal cortex distances increased with age in this depressed cohort, with D-PFC showing a trend to increase at a faster rate than D-MC. Finally, there was not a linear correlation between D-PFC and clinical antidepressant response. All subjects however, who responded were below a critical threshold of age and prefrontal cortex distance.
Limitations of the Study

There are several limitations of this study that bear on proper interpretation. The most important of these was difficulty in determining the TMS sites on the MRI scans. One method used was to place a fiducial at the site of the rTMS. When designing the study, we reasoned that this fiducial would enable a relatively precise localization of the actual site of stimulation. Using this method alone, however, would have limited an already small sample size, because only about three-fifths of the subjects studied and scanned had fiducials that could be seen on the MRI scan. Complicating things further, during scanning several of the fiducials appeared to have moved off of the scalp, resulting in a placement that no longer represented the true site of rTMS. Because of these confounding factors, we felt that using the actual fiducial to measure the distance to nearest cortex in this study was not an accurate method.

While trying to develop a more accurate method, we noticed that the prefrontal fiducial was often directly in line with a vertical (-90 degree) line from the lateral center point of the left eye. We found that the fiducial was commonly 20 slices anterior from the corpus callosum

FIGURE 3. Relationship between distance from skull to cortex and age. For all 29 subjects, motor cortex distance (circles) and prefrontal cortex distance (squares) increases with advancing age. Note that prefrontal cortex distance is often greater than motor cortex distance, and that the slope of the increase in distance with age is greater for prefrontal cortex (0.14) than for motor cortex (0.12). There is a trend for an age-related greater increase in atrophy of prefrontal cortex vs. motor cortex, but in our small cohort it is not significant. Prefrontal atrophy has been found before in patients with depression.

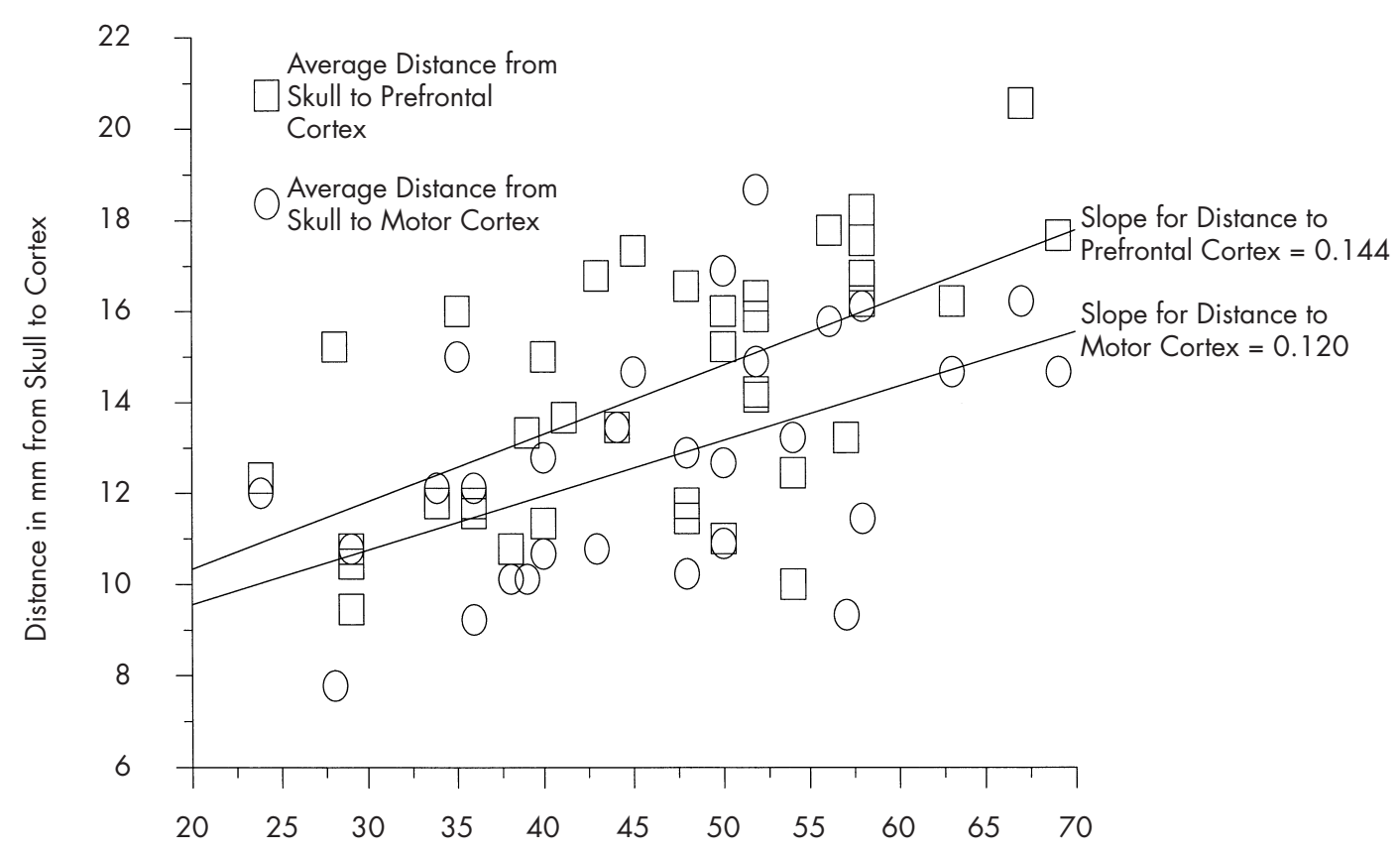

Age in Years 
and therefore adopted this as the "virtual" location of the rTMS coil to start measuring the eight scans. By performing the measurement $4 \mathrm{~mm}$ in front of and behind this chosen intersection, we averaged the scalp to cortex distance over the likely prefrontal spot. Thus, even if we did not measure directly under the actual TMS spot, we were able to obtain a distance measurement that likely represented the average distance of skull to cortex in this region. Further, this averaging system of a virtual spot enabled us to utilize all of the scans available for analysis, and the technique often correlated well with the position of the fiducial. The limitation was that we could not be sure how close to the actual coil stimulation the measurements were performed. Despite these difficulties, the standardized method of measuring eight scans and averaging the result does appear to give a rough approximation of the distance to the nearest cortex in the area stimulated. This corresponds to either the left medial frontal gyrus or the left superior frontal gyrus (Talairach coordinates: $x$ plane from -25 to $-40, y$ from 50 to $58, z$ from 20 to 40 ). Future studies with more precise fiducial placement, or even MRI phase maps of the actual magnetic field ${ }^{3}$ in all subjects, would improve on the current study.

Similarly, there was no fiducial over the motor cortex APB area where MT was actually determined. Thus, we

\section{FIGURE 4. Relationship between age and prefrontal distance separated by antidepressant responders and nonresponders. The nonresponder's regression line for distance to prefrontal cortex (D-PFC) vs. age equals $6.249+0.182^{*}$ age; $R^{2}=0.581$. The responder's regression line for D-PFC vs. age equals $12.682+0.025^{*}$ age; $R^{2}=0.013$. Note that all responders (circles) were under 55 years old and had distances less than $17 \mathrm{~mm}$. Although there was not a direct correlation between antidepressant response and prefrontal distance, these data suggest that prefrontal distance may play a role in the antidepressant response to TMS.}

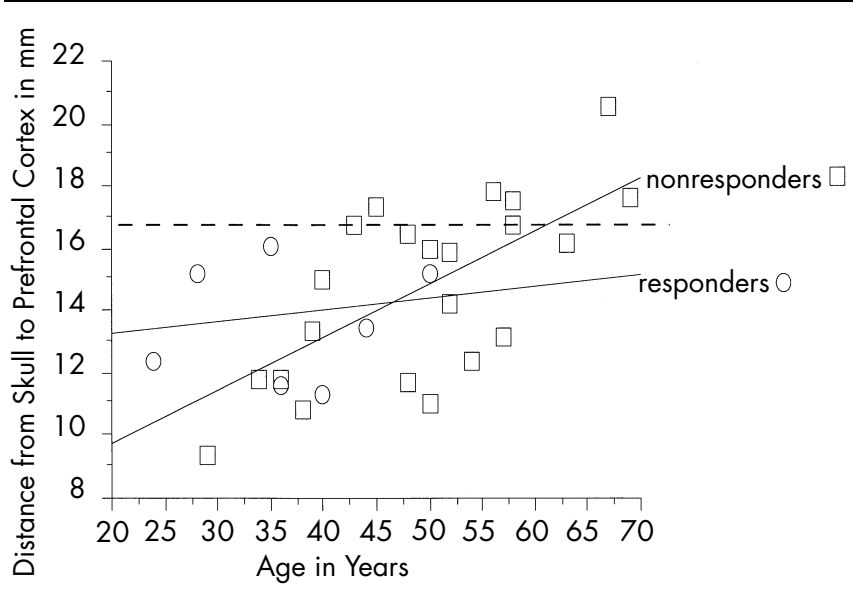

were forced to empirically determine this spot as well by measuring backward from the prefrontal site. We again measured the motor cortex distance over a relatively large area $(8 \mathrm{~mm})$ in order to compensate for the imprecision of our location. Because we measured motor cortex distance on 8 slices, the number used as a dependent variable is thus more likely a rough measure of motor cortex atrophy, rather than the exact distance from coil to motor cortex.

Despite all these factors, the robust correlation of motor threshold with distance to motor cortex is surprising, especially given these limitations in spatial location. Future studies with fiducials directly over the true site of optimum APB stimulation are needed.

The motor threshold was determined in this study by using visible movement, which is not standard practice but which our group has shown on a different machine to correspond to MT determined by motor evoked potentials. ${ }^{13}$ Future studies exploring these issues might use electrophysiologically determined MT. Similarly, our choice of the percentage change in Ham-D as the dependent variable for clinical response is relatively imprecise, but a standard practice. Future studies using other behavioral, neuroendocrine, or even brain metabolism measures might better address the correlation between distance and clinical response. Finally, the small sample size could have reduced the power of the study such that the relationship between distance to the cortex and percentage response was not statistically significant. Our sample was especially small at the extremes of age ( 5 subjects $>55$ years old) and distance to the prefrontal cortex ( 3 subjects $>17 \mathrm{~mm}$ ). On a more theoretical note, the magnetic field declines logarithmically with distance, and we tested for correlations that assumed linear relationships. Future studies with larger samples might explore whether nonlinear relationships exist between the distance variables and the other factors examined in this study. Despite these important limitations, these data provide intriguing results that will require further investigation combining rTMS and imaging.

\section{Discussion of Findings}

Motor Cortex Distance and Motor Threshold: The distance to motor cortex correlated strongly with the motor threshold, while the prefrontal distance did not (although there was a nonsignificant trend). This would imply that the distance from the coil to the nearest cortex is critical in determining the amount of energy required to depolarize the pyramidal tract neurons in the motor cortex. Another interpretation, however, is that brain atrophy by neuronal degeneration of cortical neurons may disproportionately alter the excitatory and inhibitory 
balance, requiring a higher MT. In this interpretation, the increased distance is not the most important variable and instead reflects another process that also alters the motor threshold. The current study cannot distinguish which of these, or even other, explanations lies behind the observed relationship between motor cortex distance and motor threshold.

Regardless of mechanism, a greater distance to cortex would indicate a higher motor threshold. Further studies will be needed to address this question of mechanism. In this study, the skull to motor cortex distance alone accounts for $49.7 \%$ ( $r$-correlation value) of the variance in MT across individuals. Presumably other factors such as gyral orientation and intrinsic neuronal excitation (particularly inhibition) account for the rest of the variance across individuals. This finding of the importance of distance in determining MT, if confirmed, would imply that the distance is an important variable that might be measured and used as a covariate in studies where the motor threshold is used to examine pharmacology or other questions. ${ }^{22}$

Lack of Direct Correlation Between Prefrontal Distance and Antidepressant Response: The antidepressant mechanisms of action of rTMS are unknown. Positive clinical effects have been found over both left and right prefrontal cortex, at intensities from $80 \%$ to $110 \%$ MT, and at frequencies from 0.5 to $20 \mathrm{~Hz}$. Some have suggested that the studies to date have a trend toward larger antidepressant effects with greater intensity, although this has not been directly examined. ${ }^{1}$ An assumption is made, but not formally tested, that stimulation with an intensity sufficient to cause neuronal depolarization is necessary and that low-intensity stimulation would not cause cortical cell depolarization with trans-synaptic effects. In light of these working assumptions in this new field of TMS and depression, we hypothesized that increasing skull-to-cortex distance might correlate with clinical antidepressant response. Although we failed to find a direct linear relationship, the many limitations of the current study preclude any large interpretation of this negative result. Future clinical trials in conjunction with imaging are needed to directly test the assumptions above about the antidepressant effects of TMS, intensity, and distance to prefrontal cortex.
Increase in Distance With Age: Although the motor and prefrontal measurements both increase with age and do correspond to each other, the distance to prefrontal cortex appears to increase faster with age (though not significantly) than the distance to motor cortex. This finding of greater prefrontal atrophy with age in a depressed cohort is similar to findings in other studies that have examined depressed subjects compared with agematched healthy control subjects. ${ }^{14,15}$ Although in this study MT also increased with age (though not significantly), it may be the case that there is a greater D-PFC in older depressed subjects that is not accounted for by the more modest increase in MT with age. Our finding that no individuals older than 55 years or with a prefrontal distance greater than $17 \mathrm{~mm}$ responded to rTMS is consistent with this idea, although larger studies in elderly depressed subjects are needed to directly test it. Some have suggested that older depressed patients do not respond as well to medication therapy. An agerelated variable, therefore, such as prefrontal atrophy, itself may confer a resistance to antidepressant response independent of D-PFC. Further study is indicated to understand the relationships between distance, age, and antidepressant action.

\section{Summary}

We have found that the motor threshold measurement used in TMS studies is highly dependent on the distance from cortex to skull under the TMS coil. Further, this distance increases with age, and in a depressed cohort there is prefrontal cortical atrophy that may outpace the motor cortex declines. These distances do not directly correlate with antidepressant clinical response, although TMS did not work in older subjects with large prefrontal distances. Further work combining TMS with imaging will likely expand knowledge of TMS brain effects.

The authors thank Drs. James C. Ballenger, Jeremy Young, George Arana, Eric Wassermann, Sarah Lisanby, and UlfZiemann for helpful reviews and comments. Andrew M. Speer assisted in the scanning and rTMS for the study. The National Alliance for Research on Schizophrenia and Depression and the Ted and Vada Stanley Foundation provided grants to Dr. George. An abstract of this work was presented at the New Research Session of the 152nd annual meeting of the American Psychiatric Association, Washington, DC, May 1999. ${ }^{23}$

\footnotetext{
References

1. George MS, Lisanby SH, Sackeim HA: Transcranial magnetic stimulation: applications in neuropsychiatry. Arch Gen Psychiatry 1999; 56:300-311

2. Rothwell J: Transcranial magnetic stimulation (editorial). Brain 1998; $121: 397-398$
}

3. Bohning DE, Pecheny AP, Epstein CM, et al: Mapping transcranial magnetic stimulation (TMS) fields in vivo with MRI. NeuroReport 1997; 8:2535-2538

4. Wassermann EM, Wedegaertner FR, Ziemann U, et al: Crossed 
reduction of motor cortex excitability by $1 \mathrm{~Hz}$ transcranial magnetic stimulation. Neurosci Lett 1998; 250:141-144

5. Kolbinger HM, Hoflich G, Hufnagel A, et al: Transcranial magnetic stimulation (TMS) in the treatment of major depression: a pilot study. Human Psychopharmacology 1995; 10:305-310

6. Grisaru N, Yarovslavsky U, Abarbanel J, et al: Transcranial magnetic stimulation in depression and schizophrenia. Eur Neuropsychopharmacol 1994; 4:287-288

7. Figiel GS, Epstein C, McDonald WM, et al: The use of rapid rate transcranial magnetic stimulation (rTMS) in refractory depressed patients. J Neuropsychiatry Clin Neurosci 1998; 10:2025

8. George MS, Bohning DE, Shastri A, et al: Using functional imaging to understand the neurobiological effects of TMS (abstract). CINP Abstracts 1998; st0302:101

9. Pasqual-Leone A, Valls-Sole J, Brasil-Neto JP, et al: Seizure induction and transcranial magnetic stimulation. Lancet 1992; 339:997

10. Nahas Z, Speer AM, Molloy M, et al: Role of stimulation frequency in the antidepressant effect of left prefrontal rTMS (abstract). Biol Psychiatry 1999; 45:79S-\#256

11. Loo C, Mitchell P, Sachdev P, et al: rTMS: A sham-controlled trial in medication-resistant depression (abstract). Biol Psychiatry 1998; 43:95S-\#317

12. Grunhaus L, Dannon P, Schrieber S: Effects of transcranial magnetic stimulation on severe depression: similarities with ECT (abstract). Biol Psychiatry 1998; 43:76S-\#254

13. Pridmore S, Filho JAF, Nahas Z, et al: Motor threshold in transcranial magnetic stimulation: a comparison of a neurophysiological and a visualization of movement method. J ECT 1998; $14: 25-27$
14. Coffey CE, Wilkinson WE, Weiner RD, et al: Quantitative cerebral anatomy in depression: a controlled magnetic resonance imaging study. Arch Gen Psychiatry 1993; 50:7-16

15. Krishnan KRR, McDonald WM, Escalona PR, et al: Magnetic resonance imaging of the caudate nuclei in depression. Arch Gen Psychiatry 1992; 49:553-557

16. Nahas Z, Speer AM, Lorberbaum JP, et al: Safety of rTMS: MRI scans before and after 2 weeks of daily left prefrontal rTMS for depression (abstract). Biol Psychiatry 1998; 43:95S-\#316

17. Nahas Z, Stallings LE, Speer AM, et al: Perfusion SPECT studies of rTMS on blood flow in health and depression (abstract). Biol Psychiatry 1998; 43:19S-\#63

18. Teneback C, Nahas Z, Speer AM, et al: Changes in prefrontal cortex and paralimbic activity in depression following two weeks of daily left prefrontal TMS. J Neuropsychiatry Clin Neurosci 1999; 11:426-435

19. Endicott J, Spitzer RL: A diagnostic interview: the Schedule for Affective Disorders and Schizophrenia. Arch Gen Psychiatry $1978 ; 35: 837-844$

20. Hamilton M: A rating scale for depression. J Neurol Neurosurg Psychiatry 1960; 12:56-62

21. Wassermann EM, Wang B, Zeffiro TA, et al: Locating the motor cortex on the MRI with transcranial magnetic stimulation and PET. Neuroimage 1996; 3:1-9

22. Ziemann U, Steinhoff BJ, Tergau F, et al: Transcranial magnetic stimulation: its current role in epilepsy research. Epilepsy Res 1998; 30:11-30

23. Kozel FA, Nahas Z, deBrux C, et al: Scalp to prefrontal cortex distance increases with age and might influence the antidepressant effect of left prefrontal rTMS (abstract). American Psychiatric Association annual meeting 1999; NR 181:112 